\title{
Association between Hidradenitis Suppurativa and Inflammatory Arthritis: A Systematic Review and Meta-Analysis
}

\author{
Nouf Almuhanna ${ }^{a, b}$ Alexandra Finstad $^{c}$ Raed Alhusayen ${ }^{a, b}$ \\ ${ }^{a}$ Division of Dermatology, Department of Medicine, University of Toronto, Toronto, ON, Canada; ${ }^{b}$ Division of \\ Dermatology, Department of Medicine, Sunnybrook Health Sciences Centre, Toronto, ON, Canada; ${ }^{C}$ Faculty of \\ Medicine, University of Ottawa, Ottawa, ON, Canada
}

\section{Keywords}

Acne inversa · Arthritis · Arthropathy · Comorbidity ·

Hidradenitis suppurativa

\begin{abstract}
Background: Several studies report a high prevalence of inflammatory arthritis among hidradenitis suppurativa (HS) patients. Objectives: To study the association between HS and inflammatory arthritis. Methods: The systematic review and meta-analysis were performed according to the PRISMA guidelines to identify the association between HS and inflammatory arthritis, spondyloarthritis, ankylosing spondylitis (AS), and rheumatoid arthritis (RA). Results: Seven studies were entered in the analysis, with $200,361 \mathrm{HS}$ patients and 385,599 controls. Pooled analysis illustrated a significantly increased risk of inflammatory arthritis in HS patients compared to controls (odds ratio [OR] 3.44; 95\% confidence interval [CI] 1.92-6.17). There was also a statistically significant association between HS and spondyloarthritis (OR 2.10; 95\% $\mathrm{Cl}$ 1.40-3.15), and between HS and AS (OR 1.89; 95\% CI 1.143.12). Moreover, pooled analysis showed a statistically significant association between HS and RA (OR 1.96; $95 \% \mathrm{CI}$ 1.28-2.98). Conclusions: Our findings show that HS patients
\end{abstract}

karger@karger.com www.karger.com/drm

Karger $\stackrel{\text { ' }}{5}$

GOPEN ACCESS
(C) 2021 The Author(s)

Published by S. Karger AG, Basel

This is an Open Access article licensed under the Creative Commons Attribution-NonCommercial-4.0 International License (CC BY-NC) (http://www.karger.com/Services/OpenAccessLicense), applicable to the online version of the article only. Usage and distribution for commercial purposes requires written permission. have a 3-fold increased risk of developing inflammatory arthritis. HS patients are specifically at a higher risk for spondyloarthritis, its subtype AS, and RA.

(C) 2021 The Author(s)

Published by S. Karger AG, Basel

\section{Introduction}

Hidradenitis suppurativa (HS) is a chronic, recurrent skin disorder, most commonly seen in the intertriginous regions such as the axillae and genital area [1]. It presents with tender red nodules, draining sinuses, and disfiguring scars [2]. The pathogenesis is not fully understood; studies suggest an interplay between occlusion of the pilosebaceous unit, chronic inflammation, and immune dysregulation [3]. HS was believed to be a disease limited to the skin, but current knowledge suggests that it is a systemic inflammatory disorder associated with various comorbidities such as cardiovascular disease, diabetes, inflammatory arthritis, and depression [4]. Due to chronic inflammation, HS is associated with elevated concentrations of cytokines such as tumor necrosis factor (TNF)- $\alpha$ and interleukin (IL)-17, thereby linking HS to systemic comorbidities [5]. 
Inflammatory arthritis is a group of chronic autoimmune joint disorders, including spondyloarthritis and rheumatoid arthritis (RA) [6]. Traditionally, spondyloarthritis was divided in to ankylosing spondylitis (AS), psoriatic arthritis (PsA), arthritis associated with inflammatory bowel disease, reactive arthritis, and undifferentiated spondyloarthritis; more recent classification divides spondyloarthritis into axial and peripheral arthritis [7]. Several studies report a high prevalence of inflammatory arthritis among HS patients [6], the most common type linked to HS being spondyloarthritis [8]. However, the association remains inconsistent and with a wide range of prevalence of $3.7-52.5 \%[8,9]$. The purpose of this study was to systematically investigate the association between HS and inflammatory arthritis.

\section{Materials and Methods}

\section{Search Strategy}

This systematic review and the meta-analysis were performed according to the PRISMA guidelines [10] presented in online supplementary Appendix S1 (for all online suppl. material, see www. karger.com/doi/10.1159/000514582). We searched the Cochrane Central Register, MEDLINE, Embase, and Web of Science electronic databases from their respective date of conception to 21 April 2020. The search strategy is presented in online supplementary Appendix S2.

No language restrictions were imposed. We manually examined the included studies' references to identify further pertinent articles not identified in our initial electronic database search.

\section{Study Selection and Data Extraction}

Two authors (N.A. and A.F.) independently screened titles and abstracts for eligibility. Inclusion and exclusion criteria were then implemented to the whole text for eligibility before they were included. Discrepancies were resolved by discussion among these 2 coauthors. We included cohort, case-control, and cross-sectional studies that described the association of HS with inflammatory arthritis. Case series were added in the systematic review but excluded from the meta-analysis. We included studies with both pediatric and adult HS patients. Control patients did not have HS. Exclusion criteria were case reports, abstracts, unpublished studies, and animal articles. These 2 authors then independently extracted data from the included studies using a regulated form to cross-check their results.

For each study, data extraction included: study characteristics (study type, country, author, year of publication, and data source), patient characteristics (number of patients in the HS and control groups, sex, mean age, and Hurley stage), outcome (prevalence or incidence of inflammatory arthritis in the HS and control groups), and measure of association (crude odds ratio [OR] and 95\% confidence interval $[\mathrm{CI}]$ ). We also contacted the authors of articles with missing information.

Data Analysis

We performed a random-effects meta-analysis to generate crude pooled ORs. Random effect was used because of the expect-

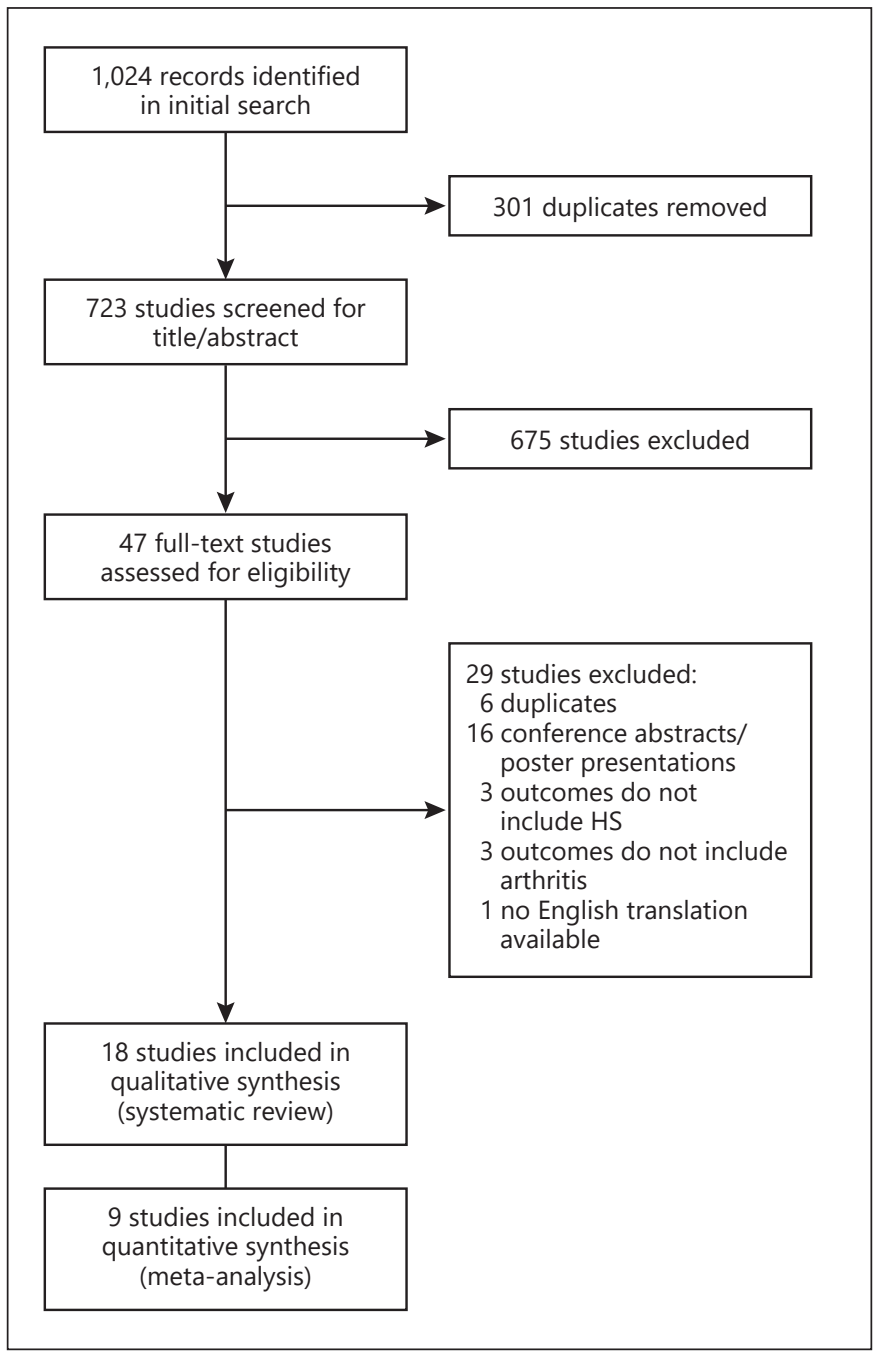

Fig. 1. Flow diagram.

ed considerable heterogeneity. Statistical heterogeneity between studies included in pooled analyses were evaluated by the Q test based on the $\chi^{2}$ test and the $I^{2}$ statistic. Values of $<25 \%$ are considered low, $25-75 \%$ moderate, and $>75 \%$ high heterogeneity [11]. $p<0.05$ was considered statistically significant.

Publication bias was examined by inspecting funnel plots. If a sufficient number of studies are included, a symmetrical plot indicates that publication bias is unlikely. All analyses were completed by Review Manager v5.3 (Cochrane Collaboration).

\section{Quality Assessment}

Two authors (N.A. and A.F.) independently completed the risk of bias assessments, using the National Institutes of Health (NIH) Quality Assessment Tool, which rates studies as being of good, fair, or poor quality. There are separate tools based on the type of study design, such as cohort and cross-sectional, case-control, and case series [12]. Discrepancies were resolved after further discussion among coauthors. 


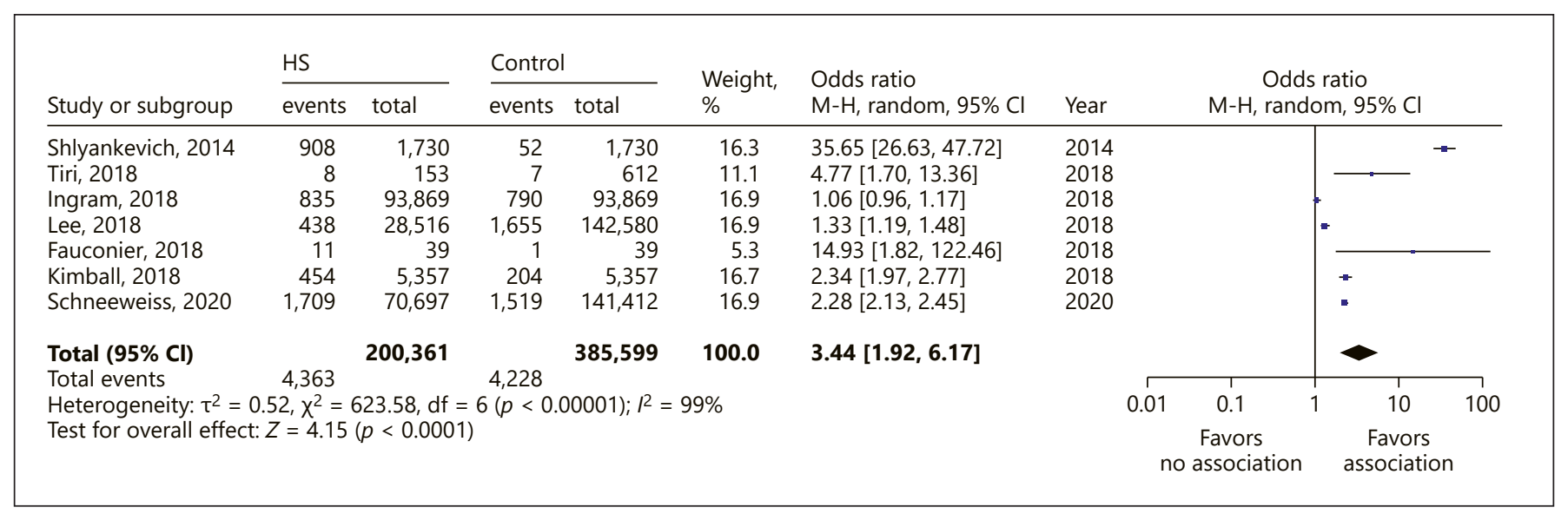

Fig. 2. Forest plot shows the association between HS and inflammatory arthritis. CI, confidence interval; crude odds ratios were used.

\section{Results}

\section{Characteristics of the Included Studies}

Our search identified 723 studies. After screening the titles and abstracts, 675 studies were excluded. We then assessed 47 full-text studies for eligibility, based on our inclusion criteria. Of these, 29 were excluded: 6 due to duplicate records, 16 conference abstracts without full text, 6 with irrelevant outcomes, and 1 because no English translation was available. Finally, 18 studies were determined as eligible for systematic review [8, 9, 13-28]. The flow chart of our search strategy is shown in Figure 1.

There were 5 cohort studies, 4 case-control studies, 6 cross-sectional studies, and 3 case series. Studies were conducted across the world: 7 in the USA, 2 in France, and 1 each in Germany, Portugal, Australia, Finland, Korea, the UK, The Netherlands, Denmark, and 1 multinational study (14 countries). With regard to participants' data, 9 studies used hospital records and 9 used electronic databases. The characteristics of the studies are presented in Table 1.

\section{Risk of Bias Assessment}

Assessing the risk of bias using the NIH Study Quality Assessment Tool, we found 8 studies with a good-quality rating, 9 with a fair rating, and 1 with a poor-quality rating due to inadequate descriptions of the cases [18] (this study was not included in any of the pooled analyses).

\section{Participant Summary}

This systematic review included 218,707 HS patients with a mean age of 38 years. A total of 200,361 HS patients entered the meta-analysis. Hurley staging for patients with HS and inflammatory arthritis was reported in 6 studies.

Pooled Analysis of the Association between HS and the Overall Risk of Developing Inflammatory Arthritis

Seven studies ( 4 case-control studies, 2 cohort studies, and 1 cross-sectional study) with 200,361 HS patients and 385,599 controls, provided data on the association between HS and the overall risk for inflammatory arthritis $[9,19-23,28]$. Pooled analysis of crude ORs illustrated a significant increase of inflammatory arthritis in HS patients compared to controls (pooled OR 3.44; 95\% CI 1.92-6.17; $p=0.0001$; Fig. 2). Substantial statistical heterogeneity was found across these 7 studies $\left(I^{2}=99 \%\right)$.

\section{Pooled Analysis of the Association between HS and} Spondyloarthritis

Four studies (2 cohort studies, 1 case-control study, and 1 cross-sectional study) with 104,609 HS patients and 289,388 controls, examined the association between HS and spondyloarthritis [20, 21, 23, 28]. As shown in Figure 3 , the association was statistically significant, with a pooled crude OR of 2.10 (95\% CI 1.40-3.15; $p=0.0003$ ). High statistical heterogeneity was found across the studies $\left(I^{2}=78 \%\right)$.

Pooled Analysis of the Association between HS and AS

Two studies (1 cohort study and 1 case-control study) with a combined 99,213 HS patients and 283,992 controls, reported the association between HS and AS [20, 28]. A significant association of $\mathrm{HS}$ with AS was observed, with a pooled OR of 1.89 (95\% CI 1.14-3.12; $p=0.01$; 


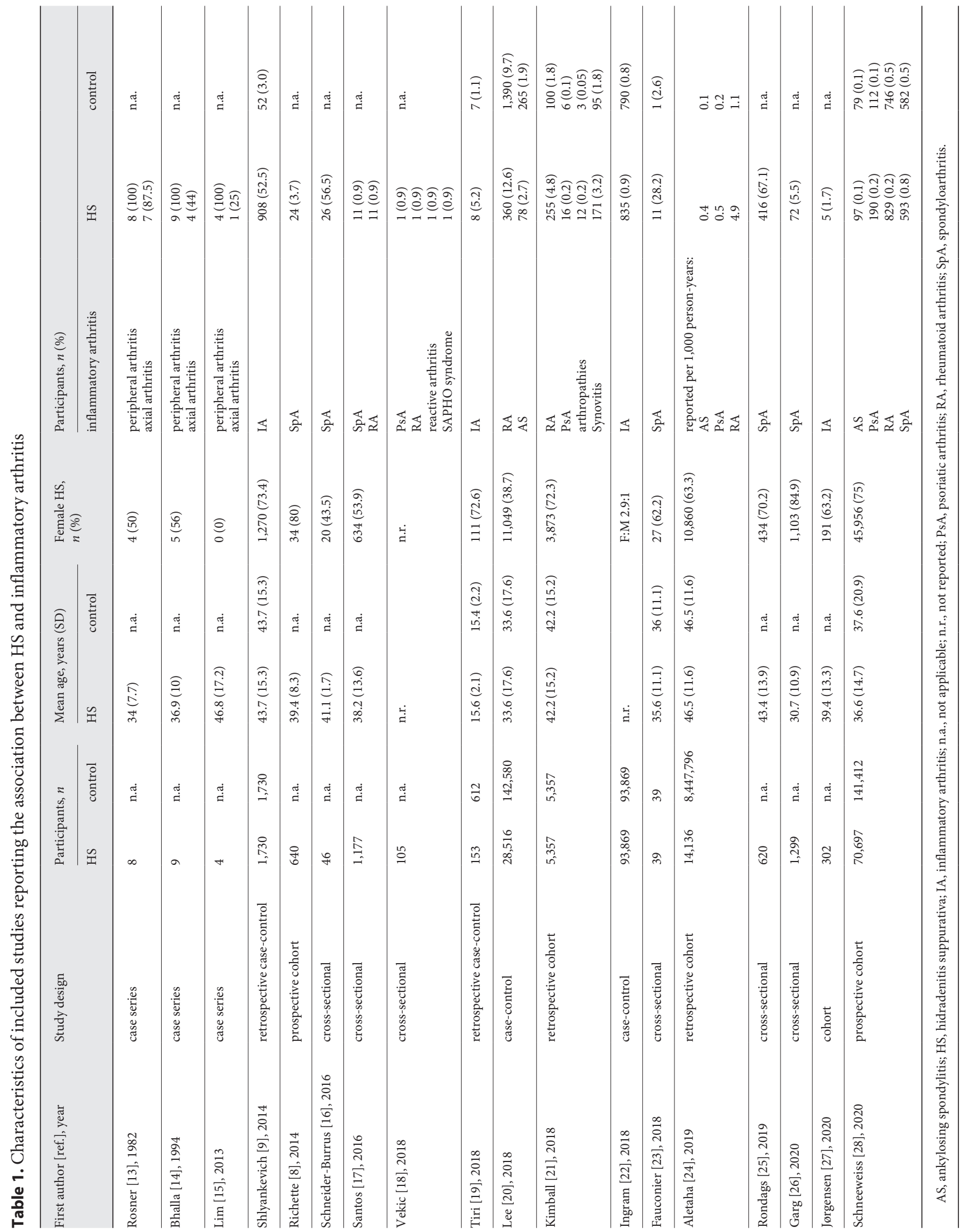




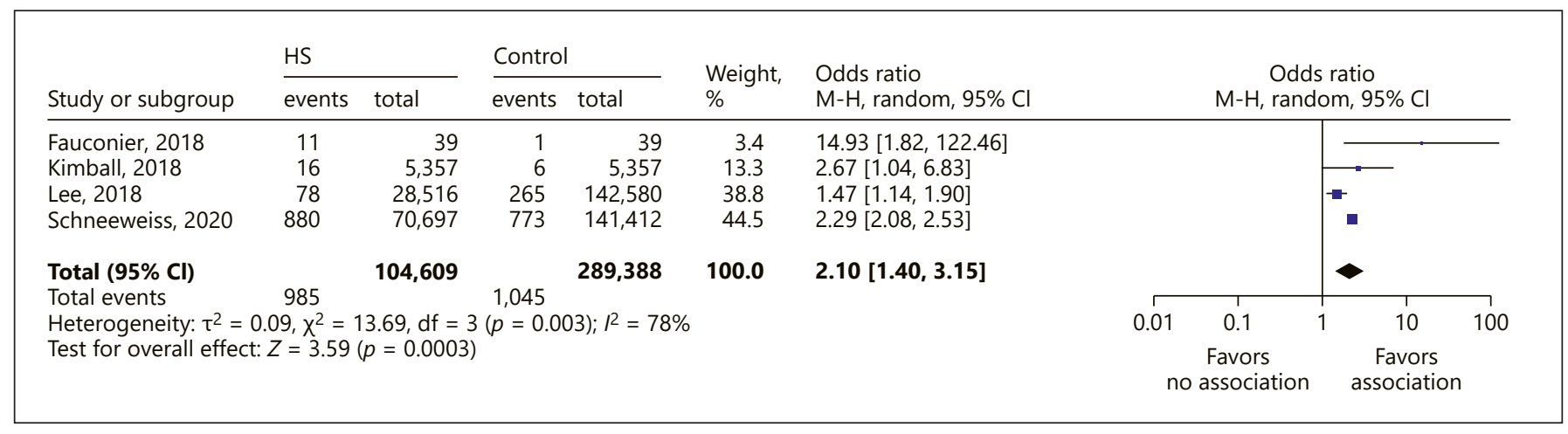

Fig. 3. Forest plots show the association between HS and spondyloarthritis. CI, confidence interval; crude odds ratios were used.

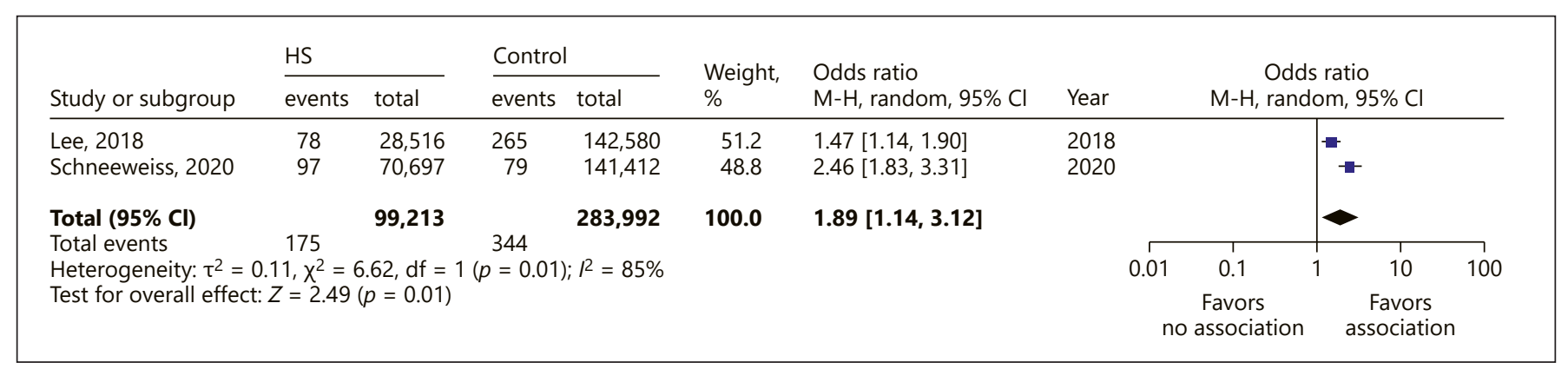

Fig. 4. Forest plots show the association between HS and ankylosing spondylitis. CI, confidence interval; crude odds ratios were used.

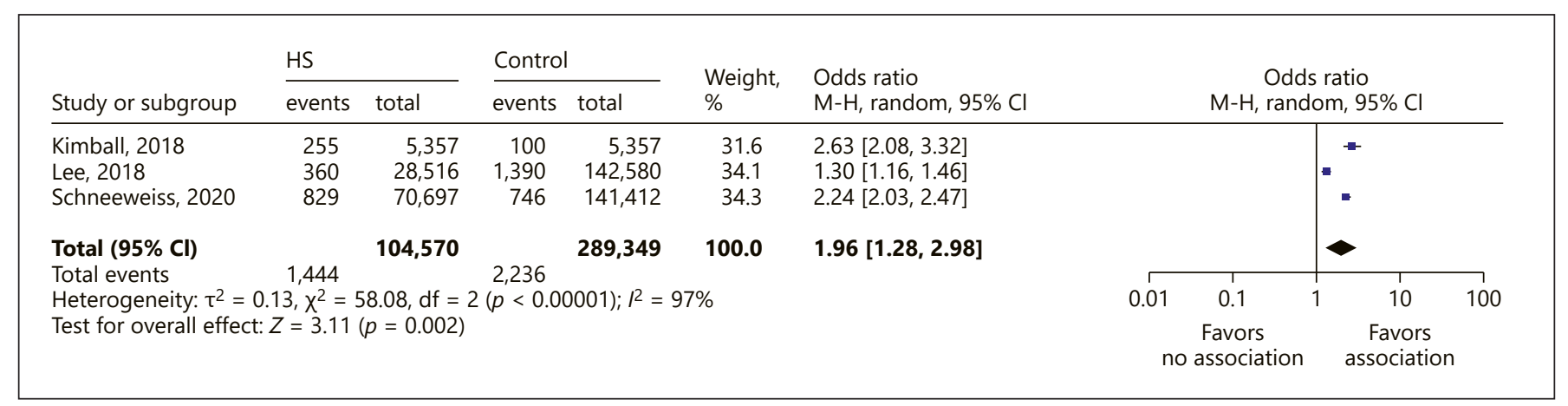

Fig. 5. Forest plot shows the association between HS and rheumatoid arthritis. CI, confidence interval; crude odds ratios were used.

Fig. 4). Statistical heterogeneity was found across these studies $\left(I^{2}=85 \%\right)$.

\section{Pooled Analysis of the Association between HS and \\ $R A$}

Three studies ( 2 cohort studies and 1 case-control study) with 104,570 HS patients and 289,349 controls, were included in the HS and RA analysis [20, 21, 28]. We found high statistical heterogeneity in these studies $\left(I^{2}=\right.$ 97\%). The pooled analysis of crude ORs demonstrated a statistically significant association (OR 1.96; 95\% CI $1.28-2.98 ; p=0.002$; Fig. 5).

Kimball et al. [21] divided HS patients into mild or severe based on severity indicators such as emergency room 
visits, biologic treatment, and skin surgery. Patients who had at least 1 of these indicators were labeled as having severe HS. They found RA in 89 (3.9\%) patients with mild HS versus 166 (5.4\%) with severe HS, and PsA in $5(0.2 \%)$ patients with mild HS versus $11(0.4 \%)$ with severe HS. They reported arthropathies in $2(0.1 \%)$ patients with mild HS versus $10(0.3 \%)$ with severe HS, and synovitis in $71(3.1 \%)$ patients with mild HS versus $100(3.3 \%)$ with severe HS.

The study by Richette et al. [8] found that spondyloarthritis was more common in patients in Hurley stage III (44\%), followed by stage II (39\%), and the least in stage I (16\%).

Given the small number of studies incorporated in the 4 meta-analyses, publication bias was not examined due to the limited power to detect asymmetry.

\section{Discussion}

This meta-analysis of 200,361 patients with HS demonstrated a significant association between HS and inflammatory arthritis. Pooled analysis indicated that patients with HS had 3.44-fold odds of having inflammatory arthritis compared to controls. When examined by type of inflammatory arthritis, patients with HS had 2.1fold odds of having spondyloarthritis, 1.96-fold odds of having RA, and 1.89-fold odds of having AS (a subtype of spondyloarthritis).

Other studies included in this systematic review but not in the meta-analysis found the prevalence of spondyloarthritis in HS patients to be higher than in the general population [8]. The prevalence of spondyloarthritis in participants ranged from 0.9 to $67.1 \%[8,16,17,25,26]$. Aletaha et al. [24] reported that the hazard ratio of HS patients developing AS was 4.4 (95\% CI 2.7-7.1), developing PsA was 2.7 (95\% CI 1.8-4.1), and developing RA was 4.6 (95\% CI 4-5.3).

Few studies commented on the association of HS severity and the presence of inflammatory arthritis, with conflicting results. Kimball et al. [21] reported a higher percentage of RA, PsA, arthropathies, and synovitis in individuals with more severe HS than in those with milder disease. Richette et al. [8] also found that inflammatory arthritis was more prevalent in patients with severe HS, with spondyloarthritis being the most predominant feature, while another study did not find any association between HS severity and the existence of spondyloarthritis [23]. Further research is required as well as investigations into a causal relationship.

Inflammatory Arthritis in HS
The exact mechanisms underlying the interaction between HS and inflammatory arthritis remain unknown. However, possible explanations include genetic susceptibility, immune dysregulation, cytokine abnormalities, and environmental factors [29].

Macrophages are the most abundant inflammatory cells in HS infiltrates and release proinflammatory cytokines including IL- $1 \beta$, TNF- $\alpha$, and IL-23, with increased levels correlating positively with disease severity $[6,29]$. Several studies have shown that smoking, a known risk factor for HS, is also a risk factor for the development and severity of spondyloarthritis and PsA [30, 31].

Across the studies, HS was reported predominantly in females, consistent with the literature. Severity of HS has been shown to be associated with male sex $[27,32]$, which may explain why comorbid inflammatory arthritis, which has been linked to HS severity $[8,21]$, has also been reported to occur predominantly in males.

Across the 3 case series, a higher percentage of $\mathrm{HS}$ patients had peripheral arthritis than axial arthritis [13-15]. It has been reported that HS patients present initially with asymmetrical peripheral arthritis whereas axial arthritis is a later manifestation $[14,15]$.

The onset of skin lesions in HS usually precedes the arthritis symptoms by 1-20 years, raising the importance of early detection of joint involvement $[14,15]$. Interestingly, although HS is considered a seronegative arthropathy, in contrast to other types of seronegative spondyloarthritis, it is not associated with the human leukocyte antigen (HLA) B27 [13, 14, 33].

It is important for clinicians treating HS patients to screen for symptoms indicative of inflammatory arthritis such as morning stiffness, lower back pain, and peripheral joint pain.

Tetracycline was shown to be effective in treating mild RA, suggesting it can be a reasonable antibiotic option for HS patients with inflammatory arthritis [34]. Other treatment options include anti-TNF biologics, sulfasalazine, systemic steroids, isotretinoin, and anakinra [15, 35-37]. However, randomized controlled trials are needed of treatment options for HS patients with comorbid inflammatory arthritis.

The limitation of our study is that our analysis incorporated a proportionately small number of studies. In addition, there was significant heterogeneity in our analysis. Regarding heterogeneity, we performed a sensitivity analysis by excluding one study at a time and observing the effect on the pooled analysis, which did not show a significant change. The possible explanation is the different definitions of arthritis between the studies due to the 
changes in the classification in the last few years. Moreover, there was no clear definition in the included studies about HS severity, which might vary between the studies and impact the prevalence of arthritis in HS patients.

This meta-analysis suggests an association between HS and inflammatory arthritis. Among the subtypes of inflammatory arthritis, HS patients are specifically at a higher risk for spondyloarthritis, its subtype AS, and RA.

Recognition of this association by dermatologists is important for early detection and can hopefully prevent further progression and complications. Patients with HS should be informed about the increased risk of inflammatory arthritis. Further research should aim to investigate the pathophysiology and evaluate effective therapies for patients with HS and inflammatory arthritis overlap.

\section{Key Message}

Hidradenitis suppurativa patients have a 3-fold increased risk of developing inflammatory arthritis.

\section{Statement of Ethics}

Ethics approval was not required, since the research is a systemic review and metanalysis of previously published studies.

\section{Conflict of Interest Statement}

The authors have no conflicts of interest to declare.

\section{Funding Sources}

There was no funding.

\section{Author Contributions}

N.A. and R.A. made contributions to study conception and design. N.A. and A.F. made contributions to the acquisition, analysis, and interpretation of data, and drafting the manuscript. R.A. revised the manuscript critically and approved the final version. N.A. agreed to be accountable for all aspects of the work in ensuring that questions related to the accuracy or integrity of any part of the work are appropriately investigated and resolved. All authors read and approved the final manuscript. All authors agreed on the order in which their names are listed.

\section{References}

1 Phan K, Charlton O, Smith SD. Hidradenitis suppurativa and diabetes mellitus: updated systematic review and adjusted meta-analysis. Clin Exp Dermatol. 2019 Jun;44(4):e126-32.

2 Oskardmay AN, Miles JA, Sayed CJ. Determining the optimal dose of infliximab for treatment of hidradenitis suppurativa. J Am Acad Dermatol. 2019 Sep;81(3):702-8.

3 Alikhan A, Sayed C, Alavi A, Alhusayen R, Brassard A, Burkhart C, et al. North American clinical management guidelines for hidradenitis suppurativa: A publication from the United States and Canadian Hidradenitis Suppurativa Foundations: Part I: Diagnosis, evaluation, and the use of complementary and procedural management. J Am Acad Dermatol. 2019 Jul;81(1):76-90.

4 Reddy S, Strunk A, Garg A. Comparative overall comorbidity burden among patients with hidradenitis suppurativa. JAMA Dermatol. 2019 Jul;155(7):797-802.

5 van der Zee HH, de Ruiter L, van den Broecke DG, Dik WA, Laman JD, Prens EP. Elevated levels of tumour necrosis factor (TNF)- $\alpha$, interleukin (IL)-1 $\beta$ and IL-10 in hidradenitis suppurativa skin: a rationale for targeting TNF- $\alpha$ and IL-1 $\beta$. Br J Dermatol. 2011 Jun; 164(6):1292-8.

6 Dauden E, Lazaro P, Aguilar MD, Blasco AJ, Suarez C, Marin I, et al. Recommendations for the management of comorbidity in hi- dradenitis suppurativa. J Eur Acad Dermatol Venereol. 2018 Jan;32(1):129-44.

7 Rudwaleit M, van der Heijde D, Landewé R, Akkoc N, Brandt J, Chou CT, et al. The Assessment of SpondyloArthritis International Society classification criteria for peripheral spondyloarthritis and for spondyloarthritis in general. Ann Rheum Dis. 2011 Jan;70(1):2531.

8 Richette P, Molto A, Viguier M, Dawidowicz K, Hayem G, Nassif A, et al. Hidradenitis suppurativa associated with spondyloarthritisresults from a multicenter national prospective study. J Rheumatol. 2014 Mar;41(3):4904.

9 Shlyankevich J, Chen AJ, Kim GE, Kimball AB. Hidradenitis suppurativa is a systemic disease with substantial comorbidity burden: a chart-verified case-control analysis. J Am Acad Dermatol. 2014 Dec;71(6):1144-50.

10 Moher D, Liberati A, Tetzlaff J, et al. The PRISMA 2009 Flow Diagram. The PRISMA statement. PLoS Med. 2009;6(7):e1000097.

11 Higgins JP, Thompson SG. Quantifying heterogeneity in a meta-analysis. Stat Med. 2002 Jun;21(11):1539-58.

12 U.S. Department of Health and Human Services, National Institutes of Health, National Heart Lung and Blood Institute (NIH). Assessing cardiovascular risk - Systematic evidence review from the risk assessment work group. Available from: https://www.nhlbi. nih.gov/health-topics/study-quality-assessment-tools.

13 Rosner IA, Richter DE, Huettner TL, Kuffner GH, Wisnieski JJ, Burg CG. Spondyloarthropathy associated with hidradenitis suppurative and acne conglobata. Ann Intern Med. 1982 Oct;97(4):520-5.

14 Bhalla R, Sequeira W. Arthritis associated with hidradenitis suppurativa. Ann Rheum Dis. 1994 Jan;53(1):64-6.

15 Lim DT, James NM, Hassan S, Khan MA Spondyloarthritis associated with acne conglobata, hidradenitis suppurativa and dissecting cellulitis of the scalp: a review with illustrative cases. Curr Rheumatol Rep. 2013 Aug; 15(8):346.

16 Schneider-Burrus S, Witte-Haendel E, Christou D, Rigoni B, Sabat R, Diederichs G. High prevalence of back pain and axial spondyloarthropathy in patients with hidradenitis suppurativa. Dermatology. 2016;232(5):606-12.

17 Santos JV, Lisboa C, Lanna C, Costa-Pereira A, Freitas A. Hospitalisations with hidradenitis suppurativa: an increasing problem that deserves closer attention. Dermatology. 2016; 232(5):613-8.

18 Vekic DA, Frew J, Cains GD. Hidradenitis suppurativa, a review of pathogenesis, associations and management. Part 1. Australas J Dermatol. 2018 Nov;59(4):267-77. 
19 Tiri H, Jokelainen J, Timonen M, Tasanen K, Huilaja L. Somatic and psychiatric comorbidities of hidradenitis suppurativa in children and adolescents. J Am Acad Dermatol. 2018 Sep;79(3):514-9.

20 Lee JH, Kwon HS, Jung HM, Kim GM, Bae JM. Prevalence and comorbidities associated with hidradenitis suppurativa in Korea: a nationwide population-based study. J Eur Acad Dermatol Venereol. 2018 Oct;32(10):1784-90.

21 Kimball AB, Sundaram M, Gauthier G, Guérin A, Pivneva I, Singh R, et al. The comorbidity burden of hidradenitis suppurativa in the United States: A claims data analysis. Dermatol Ther (Heidelb). 2018 Dec;8(4): 557-69.

22 Ingram JR, Jenkins-Jones S, Knipe DW, Morgan CL, Cannings-John R, Piguet V. Population-based Clinical Practice Research Datalink study using algorithm modelling to identify the true burden of hidradenitis suppurativa. Br J Dermatol. 2018 Apr;178(4): 917-24.

23 Fauconier M, Reguiai Z, Barbe C, Colosio A, Eschard JP, Salmon JH, et al. Association between hidradenitis suppurativa and spondyloarthritis. Joint Bone Spine. 2018 Oct;85(5): 593-7.

24 Aletaha D, Epstein AJ, Skup M, Zueger P, Garg V, Panaccione R. Risk of developing additional immune-mediated manifestations: A retrospective matched cohort study. Adv Ther. 2019 Jul;36(7):1672-83.
25 Rondags A, van Straalen KR, Arends S, van der Zee HH, Prens EP, Spoorenberg A, et al. High prevalence of clinical spondyloarthritis features in patients with hidradenitis suppurativa. J Am Acad Dermatol. 2019 Feb;80(2): 551-554.e1.

26 Garg A, Neuren E, Cha D, Kirby JS, Ingram JR, Jemec GB, et al. Evaluating patients' unmet needs in hidradenitis suppurativa: Results from the Global Survey Of Impact and Healthcare Needs (VOICE) Project. J Am Acad Dermatol. 2020 Feb;82(2):36676.

27 Jørgensen AR, Yao Y, Ghazanfar MN, Ring HC, Thomsen SF. Burden, predictors and temporal relationships of comorbidities in patients with hidradenitis suppurativa: a hospital-based cohort study. J Eur Acad Dermatol Venereol. 2020 Mar;34(3):56573.

28 Schneeweiss MC, Kim SC, Schneeweiss S, Rosmarin D, Merola JF. Risk of inflammatory arthritis after a new diagnosis of hidradenitis suppurativa. JAMA Dermatol. 2020 Mar; 156(3):342-5.

29 Shah A, Alhusayen R, Amini-Nik S. The critical role of macrophages in the pathogenesis of hidradenitis suppurativa. Inflamm Res. 2017 Nov;66(11):931-45.

30 Chung HY, Machado P, van der Heijde D, D'Agostino MA, Dougados M. Smokers in early axial spondyloarthritis have earlier disease onset, more disease activity, inflamma- tion and damage, and poorer function and health-related quality of life: results from the DESIR cohort. Ann Rheum Dis. 2012 Jun; 71(6):809-16.

31 Li W, Han J, Qureshi AA. Smoking and risk of incident psoriatic arthritis in US women. Ann Rheum Dis. 2012 Jun;71(6):804-8.

32 Schrader AM, Deckers IE, van der Zee HH, Boer J, Prens EP. Hidradenitis suppurativa: a retrospective study of 846 Dutch patients to identify factors associated with disease severity. J Am Acad Dermatol. 2014 Sep;71(3): 460-7.

33 Ellis BI, Shier CK, Leisen JJ, Kastan DJ, McGoey JW. Acne-associated spondylarthropathy: radiographic features. Radiology. 1987 Feb;162(2):541-5.

34 Ogrendik M. Antibiotics for the treatment of rheumatoid arthritis. Int J Gen Med. 2013 Dec;7:43-7.

35 Libow LF, Friar DA. Arthropathy associated with cystic acne, hidradenitis suppurativa, and perifolliculitis capitis abscedens et suffodiens: treatment with isotretinoin. Cutis. 1999 Aug;64(2):87-90.

36 Thein M, Hogarth MB, Acland K. Seronegative arthritis associated with the follicular occlusion triad. Clin Exp Dermatol. 2004 Sep; 29(5):550-2.

37 Scheinfeld N. Treatment of coincident seronegative arthritis and hidradentis supprativa with adalimumab. J Am Acad Dermatol. 2006 Jul;55(1):163-4. 